\title{
A high consumption of tomato and lycopene is associated with a lower risk of cancer mortality: results from a multi-ethnic cohort
}

\author{
Mohsen Mazidi ${ }^{1, *} \uparrow$, Gordon A Ferns ${ }^{2}$ and Maciej Banach $3,4,5$ \\ 'Department of Biology and Biological Engineering, Food and Nutrition Science, Chalmers University of Technology, \\ Gothenburg, Sweden: ${ }^{2}$ Division of Medical Education, Brighton and Sussex Medical School, University of Brighton, \\ Brighton, UK: ${ }^{3}$ Department of Hypertension, Chair of Nephrology and Hypertension, Medical University of Lodz, Lodz, \\ Poland: " Polish Mother's Memorial Hospital Research Institute (PMMHRI), Lodz, Poland: ${ }^{5}$ Cardiovascular Research \\ Centre, University of Zielona Gora, Zielona Gora, Poland
}

Submitted 13 June 2018: Final revision received 15 May 2019: Accepted 24 June 2019: First published online 27 February 2020

\begin{abstract}
Objective: We investigated the association between the consumption of tomato and lycopene and cancer mortality among US adults.

Design: Prospective.

Setting: The National Health and Nutrition Examination Survey (1999-2010).

Participants: Participants with estimated dietary data on tomato and lycopene consumption were included. Outcome data up until 31 December 2011 were also ascertained. Cox proportional hazard regression models were used to relate baseline tomato and lycopene consumption with cancer mortality. We conducted a competing-risk survival analysis to account for deaths from other causes.

Results: Adjusted Cox models showed that tomato and lycopene intake were inversely related (hazard ratio $(95 \% \mathrm{CI}))$ to cancer mortality: $0.86(0.81,0.92)$ and $0.79(0.74,0.82)$, respectively. In the adjusted competing-risk models, the sub-hazard ratios $(95 \% \mathrm{CI})$ were $0.89(0.83,0.94)$ and $0.82(0.78,0.86)$ for cancer mortality for tomato and lycopene intake, respectively. No significant interaction was found for the association between tomato and lycopene consumption and cancer mortality while comparing older (aged $>50$ years) $v$. younger adults $\left(P_{\text {interaction }}>0.173\right.$ for all) and obese $v$. non-obese $\left(P_{\text {interaction }}>0.352\right.$ for all). Conclusions: Our results demonstrate the potential beneficial effects of a high dietary intake of tomato and lycopene on cancer death. Further prospective studies are needed to explore the association.
\end{abstract}

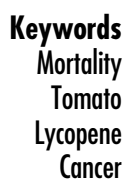

According to the American Cancer Society, 7.6 million people (representing $13 \%$ of all deaths) worldwide died from malignant neoplasms in 2008, a figure predicted to rise to 12 million by $2030^{(1)}$. Given the burden of the morbidity and mortality from cancers, reducing their risk with primary prevention strategies that may reduce disease burden could have an important impact on public health. Consumption of fruits and vegetables may act by inhibiting the multistep carcinogenic process by several mechanisms, for example via their antioxidant properties, cell-cycle arrest or pro-vitamin A activity ${ }^{(2-4)}$.

Lycopene is abundant in US diets. This bioactive deep-red pigment is naturally synthesized by fruits and

†Present address: Department of Twin Research and Genetic Epidemiology, King's College London, St Thomas' Hospital, Strand, London SE1 7EH, UK. vegetables and is the most abundant carotenoid in red tomatoes and tomato-based products, including ketchup, tomato juice and pizza sauce ${ }^{(5,6)}$. Tomatoes contain moderate amounts of $\alpha$ - and $\beta$-carotene and vitamin $C$, which have antioxidant and immunostimulatory properties ${ }^{(7,8)}$. The strong antioxidant properties of lycopene and other constituents of tomato have a potential role as regulators of gene function. Lycopene has also been shown to participate in the modulation of hormone and immune activity ${ }^{(9)}$ and in the metabolism of carcinogens ${ }^{(10)}$. Thus, dietary lycopene and tomato may exert their chemopreventive effects in the setting of numerous diseases, including some cancers $^{(11)}$. These findings have triggered interest in the effects of lycopene and tomato consumption on health outcomes. However, some studies have not confirmed the protective effects of lycopene and tomato on cancer 
risk $^{(12-16)}$, which has led to uncertainty in relation to the association of lycopene/tomato consumption and cancer risk.

Therefore, given to paucity of studies and the high burden of cancer mortality, we sought to design a prospective study to examine the association of tomato and lycopene consumption with cancer mortality, by reviewing the dietary data from a nationally representative sample of US adults. We hypothesized that a higher consumption of tomato and lycopene would be associated with a lower risk of cancer mortality.

\section{Methods}

\section{Population}

The current study used data from the prospective US National Health and Nutrition Examination Survey (NHANES). The National Center for Health Statistics Research Ethics Review Board approved the underlying protocol and written informed consent was obtained from all participants. The current study is based on the analysis of data from 2-year NHANES survey cycles between 1999 and 2010, restricted to participants aged $\geq 20$ years. Details on the NHANES laboratory/medical technologists' procedures and anthropometry procedures are described elsewhere ${ }^{(17,18)}$. Trained interviewers collected the demographic, socio-economic, dietary and health-related information of participants using questionnaires administered during home visits. Clinical examination and dietary assessment were conducted by skilled personnel in mobile examination centres ${ }^{(19)}$. All procedures were carried out in accordance with relevant approved guidelines and regulations ${ }^{(17,20-22)}$.

Dietary intake was assessed from a $24 \mathrm{~h}$ recall obtained by a trained interviewer with the use of a computerassisted dietary interview system with standardized probes, namely the US Department of Agriculture's Automated Multiple-Pass Method ${ }^{(23,24)}$. Briefly, the type and quantity of all foods and beverages consumed in a single $24 \mathrm{~h}$ period before the dietary interview (from midnight to midnight) were collected with the use of the Automated Multiple-Pass Method, which is designed to facilitate the collection of complete and accurate data while reducing respondent burden ${ }^{(24,25)}$. The US Department of Agriculture's Food and Nutrient Database for Dietary Studies was used to determine the nutrient content of foods.

A digital scale was used to measure body weight to the nearest $100 \mathrm{~g}$ and a fixed stadiometer to measure height to the nearest $1 \mathrm{~mm}$. BMI was calculated as body weight in kilograms divided by the square of height in metres. Smoking was based on self-report. The poverty to income variable is an index for the ratio of family income to poverty. The Department of Health and Human Services' poverty guidelines were used as the poverty measure to calculate this index ${ }^{(22)}$. Three blood pressure readings (including systolic and diastolic blood pressure) were recorded by a physician using a mercury sphygmomanometer and the average of the three measurements was obtained. Blood pressure was measured in the right arm unless otherwise specified. A blood specimen was drawn from the participant's antecubital vein. Fasting blood glucose was measured by the hexokinase method using a Roche/Hitachi 911 Analyzer and a Roche Modular P Chemistry Analyzer ${ }^{(26)}$. Diabetes was diagnosed as a selfreported history of diabetes or fasting plasma glucose $\geq 126 \mathrm{mg} / \mathrm{dl}^{(27)}$. Hypertension was diagnosed in individuals with systolic blood pressure $\geq 140 \mathrm{mmHg}$ and/or diastolic blood pressure $\geq 90 \mathrm{mmHg}^{(28)}$. Other laboratory test details are available in the NHANES Laboratory/Medical Technologists' Procedures Manual ${ }^{(19)}$.

\section{Mortality}

The de-identified and anonymized data of NHANES 1999-2010 participants were linked to longitudinal Medicare and mortality data using the NHANES-assigned sequence number. Follow-up mortality data were available from the date of survey participation until 31 December 2011. We examined mortality due to cancer (C00-C97). Cause of death was determined using the 10th revision of the International Classification of Diseases $^{(29)}$.

\section{Statistical analysis}

Analyses were conducted according to the guidelines set by the Centers for Disease Control and Prevention for analysis of the NHANES data set, accounting for the masked variance and using the Centers' suggested weighting methodology ${ }^{(30,31)}$. Continuous and categorical demographic variables were compared across tertiles of tomato and lycopene consumption using ANOVA and $\chi^{2}$ tests, respectively.

Multivariable Cox proportional hazards were applied to determine the hazard ratios (HR) and $95 \%$ CI of mortality for each tertile (T) of tomato and lycopene consumption, with the lowest tertile (T1) always used as reference. To derive the $\mathrm{HR}$ and $95 \% \mathrm{CI}$, we used two different models: model 1 adjusted for age, gender, race, education, marital status, poverty to income ratio, total energy intake, physical activity, smoking and alcohol consumption; and model 2 adjusted for age, gender, race, education, marital status, poverty to income ratio, total energy intake, physical activity, smoking, alcohol consumption, BMI, dietary fat, C-reactive protein, carbohydrates, dietary fibre, meat, BMI, hypertension and diabetes. Fine-Gray competing-risk regression models, with adjustment, were used to assess the risk of cancer mortality where all-cause mortality was considered a competing event ${ }^{(32)}$. A twosided $P$ value of $<0.05$ was deemed significant. 
Data were analysed using the complex sample module of the statistical software package IBM SPSS ${ }^{\circledR}$ Statistics version 22.0 .

\section{Results}

Overall, 22835 participants were included, with a mean age of 47.8 years, comprising $48.9 \%$ men. Demographic characteristics of the participants according to categories of tomato and lycopene consumption are shown in Table 1. Individuals with the highest tomato intake (T3, 1.8 cups consumed/d) were significantly younger compared with those with the least tomato consumption (T1, 0.02 cups consumed/d; $43.4 v 49.5$ years, respectively, $P<0.001$, Table 1). Men comprised the majority of those in the highest tomato consumption group $(53.2 \%$, $P<0.001)$, whereas females comprised the highest percentage in the lowest category $(53.4 \%, P<0.001$, Table 1). In the category of individuals with the highest tomato intake (T3), the ethnic distribution was as follows: non-Hispanic White (52.0 \%), non-Hispanic Black (23.9\%) and Mexican-American $(12.7 \%, P<0.001$, Table 1$)$. The majority of individuals with an educational attainment of 'more than high school' (49.2\%) were in the category with highest tomato consumption (T3, 1.8 cups consumed/d), while most of those with 'less than high school' (30.2\%) education were in the lowest category (T1, 0.02 cups consumed/d, Table 1). In case of the lycopene intake, figures and trends were the same as for tomato intake (Table 1).

During the median follow-up of 76.4 months, a total 3469 deaths were recorded. Of these deaths, 796 were due to cancers. We found that there was a lower rate of mortality from cancer in the group with the highest (T3) tomato and lycopene intake ( 42.5 and $45.9 \%$, respectively) compared with the group with the lowest tomato and lycopene consumption (T1, $P<0 \cdot 001)$.

Using model 1 (adjusted for age, gender, race, education, marital status, poverty to income ratio, total energy intake, physical activity, smoking and alcohol consumption), there was a negative (27\%) association between tomato consumption and cancer mortality $(\mathrm{HR}=0.73$, $95 \%$ CI 0.64, 0.84); for model 2 (adjusted for age, gender, race, education, marital status, poverty to income ratio, total energy intake, physical activity, smoking, alcohol consumption, BMI, dietary fat, C-reactive protein, carbohydrates, dietary fibre, meat, BMI, hypertension and diabetes), the association was attenuated but still significant ( $\mathrm{HR}=0.86,95 \% \mathrm{CI} 0 \cdot 81,0.92$ ). As for lycopene intake, we found an inverse association between lycopene consumption and cancer mortality in model $1(\mathrm{HR}=0 \cdot 68,95 \% \mathrm{CI}$ $0 \cdot 60,0 \cdot 79)$ and this association remained even after adjustment for additional confounders (model 2) as a $21 \%$ lower

Table 1 Characteristics of the study participants according to tomato and lycopene consumption: US adults aged $\geq 20$ years, National Health and Nutrition Examination Survey (1999-2010)

\begin{tabular}{|c|c|c|c|c|c|c|c|c|c|c|c|c|c|c|}
\hline & \multicolumn{6}{|c|}{ Tomato consumption tertile } & \multicolumn{7}{|c|}{ Lycopene consumption tertile } & \multirow[b]{3}{*}{$P_{\text {trend }}$} \\
\hline & \multicolumn{2}{|c|}{$\begin{array}{c}\text { T1 } \\
(0.02 \text { cups } \\
\text { consumed/d) }\end{array}$} & \multicolumn{2}{|c|}{$\begin{array}{c}\text { T2 } \\
\text { (0.3 cups } \\
\text { consumed/d) }\end{array}$} & \multicolumn{2}{|c|}{$\begin{array}{c}\text { T3 } \\
(1.8 \text { cups } \\
\text { consumed/d) }\end{array}$} & \multirow[b]{2}{*}{$P_{\text {trend }}$} & \multicolumn{2}{|c|}{$\begin{array}{c}\text { T1 } \\
(485(265- \\
796) \mu \mathrm{g})^{\star}\end{array}$} & \multicolumn{2}{|c|}{$\begin{array}{c}\text { T2 } \\
(1605(1029- \\
2685) \mu \mathrm{g})^{*}\end{array}$} & \multicolumn{2}{|c|}{$\begin{array}{c}\text { T3 } \\
(9365(5934- \\
12463) \mu \mathrm{g})^{*}\end{array}$} & \\
\hline & $\begin{array}{c}\text { Mean or } \\
\%\end{array}$ & SE & $\begin{array}{c}\text { Mean or } \\
\%\end{array}$ & $\mathrm{SE}$ & $\begin{array}{c}\text { Mean or } \\
\%\end{array}$ & SE & & $\begin{array}{c}\text { Mean or } \\
\%\end{array}$ & SE & $\begin{array}{c}\text { Mean or } \\
\%\end{array}$ & SE & $\begin{array}{c}\text { Mean or } \\
\%\end{array}$ & SE & \\
\hline $\begin{array}{l}\text { Age (years), mean } \\
\text { and SE }\end{array}$ & $49 \cdot 5$ & 0.2 & $46 \cdot 3$ & 0.1 & $43 \cdot 4$ & 0.2 & $<0.001$ & $50 \cdot 6$ & 0.2 & $47 \cdot 2$ & 0.2 & $44 \cdot 8$ & $0 \cdot 2$ & $<0.001$ \\
\hline \multicolumn{15}{|l|}{ Gender (\%) } \\
\hline Men & $46 \cdot 6$ & - & $45 \cdot 8$ & - & $53 \cdot 2$ & - & $<0.001$ & $46 \cdot 5$ & - & $45 \cdot 2$ & - & $53 \cdot 6$ & - & $<0.001$ \\
\hline Women & 53.4 & - & $54 \cdot 2$ & - & $46 \cdot 8$ & - & & 53.5 & - & 54.7 & - & $46 \cdot 4$ & - & \\
\hline \multicolumn{15}{|l|}{ Race/ethnicity (\%) } \\
\hline Mexican-American & $19 \cdot 4$ & - & $17 \cdot 3$ & - & $12 \cdot 7$ & - & $<0.001$ & $21 \cdot 6$ & - & $19 \cdot 4$ & - & 14.5 & - & $<0.001$ \\
\hline Non-Hispanic White & $46 \cdot 6$ & - & $48 \cdot 1$ & - & $52 \cdot 0$ & - & & $47 \cdot 2$ & - & 49.5 & - & $54 \cdot 3$ & - & \\
\hline Non-Hispanic Black & $19 \cdot 4$ & - & $22 \cdot 8$ & - & 23.9 & - & & $20 . \overline{7}$ & - & $21 \cdot 3$ & - & $22 \cdot 4$ & - & \\
\hline \multicolumn{15}{|l|}{ Marital status (\%) } \\
\hline Married & 51.4 & - & $53 \cdot 2$ & - & $52 \cdot 3$ & - & $<0.001$ & $52 \cdot 2$ & - & $53 \cdot 3$ & - & $52 \cdot 3$ & - & $<0.001$ \\
\hline Divorced & $10 \cdot 7$ & - & $10 \cdot 2$ & - & $11 \cdot 1$ & - & & 11.2 & - & $10 \cdot 2$ & - & $11 \cdot 2$ & - & \\
\hline Never married & $17 \cdot 6$ & - & $9 \cdot 3$ & - & $20 \cdot 4$ & - & & 19.9 & - & $17 \cdot 4$ & - & 18.5 & - & \\
\hline \multicolumn{15}{|l|}{ Education status (\%) } \\
\hline $\begin{array}{l}\text { Less than high } \\
\text { school }\end{array}$ & $30 \cdot 2$ & - & $28 \cdot 0$ & - & $27 \cdot 4$ & - & $<0.001$ & $28 \cdot 4$ & - & $24 \cdot 1$ & - & $26 \cdot 7$ & - & $<0.001$ \\
\hline $\begin{array}{l}\text { Completed high } \\
\text { school }\end{array}$ & $24 \cdot 3$ & - & $23 \cdot 6$ & - & $24 \cdot 7$ & - & & $25 \cdot 6$ & - & $23 \cdot 1$ & - & $26 \cdot 0$ & - & \\
\hline $\begin{array}{l}\text { More than high } \\
\text { school }\end{array}$ & $46 \cdot 4$ & - & $47 \cdot 2$ & - & $49 \cdot 2$ & - & & $47 \cdot 5$ & - & $45 \cdot 0$ & - & $48 \cdot 1$ & - & \\
\hline
\end{tabular}

Values are expressed as means and their standard errors or as percentages. Groups across the tertile were compared by either the $\chi^{2}$ test or ANOVA

*Lycopene consumption is expressed as median (25th-75th percentile). 
risk $\quad(\mathrm{HR}=0.79, \quad 95 \%$ CI $0.74, \quad 0.82)$. Figures for the adjusted competing-risk models, sub-hazard ratios, were (tomato: $\mathrm{HR}=0.89,95 \%$ CI 0.83, 0.94; lycopene: $\mathrm{HR}=0.82,95 \% \mathrm{CI} 0.78,0.86$ ) for individuals with the highest tomato and lycopene intake.

In model 2 (adjusted for age, gender, race, education, marital status, poverty to income ratio, total energy intake, physical activity, smoking, alcohol consumption, BMI, dietary fat, C-reactive protein, carbohydrates, dietary fibre, meat, BMI, hypertension and diabetes), we found that there was no difference between non-obese and obese $\left(\mathrm{BMI} \geq 30 \mathrm{~kg} / \mathrm{m}^{2}\right)$ participants in the case of the association of both tomato and lycopene with risk of cancer mortality ( $P_{\text {interaction }}>0.35$ for all). Further, no interaction of tomato and lycopene consumption with risk of cancer mortality in regard to older (aged $>50$ years) and younger adults ( $P_{\text {interaction }}>0 \cdot 17$ for all) was observed.

\section{Discussion}

By using data from a nationally representative sample of US adults, we aimed to evaluate the impact of tomato and lycopene consumption on cancer mortality. In the adjusted models there was an inverse association of cancer mortality with both tomato and lycopene intake (i.e. higher intake of tomato and lycopene was associated with a lower risk of mortality); this association was robust after further adjustments for clinical, dietary and anthropometrical confounders and remained significant using competing-risk analyses, where deaths from other causes were positioned as competitors to cancer mortality.

During the last several decades, studies have highlighted the beneficial effects of high dietary tomato and lycopene intake, being associated with a lower cancer risk $^{(33)}$. High intake of tomatoes has consistently been found to be associated with reduced risk of digestive-tract (especially stomach, colon and rectal) cancers in a casecontrol study from Italy, where cases were patients with histologically confirmed cancers of the oral cavity, pharynx, oesophagus, stomach, colon and rectum, and controls were patients with unrelated conditions ${ }^{(34)}$. Lycopene is one of the most potent antioxidants and the most abundant carotenoid in human plasma, and it is assumed to be one of the active compounds responsible for the health benefits of tomato ${ }^{(35)}$. An inverse association between lycopene (intake or serum level) and breast cancer risk has been reported by some investigators in epidemiological investigations $^{(33,36,37)}$. Intake of lycopene was inversely related to the risk of prostate cancer ${ }^{(33,38,39)}$. A risk reduction of almost $35 \%$ was observed for a consumption frequency of ten or more servings of tomato products per week, and the protective effects were even stronger for more advanced or aggressive prostate cancer ${ }^{(33,37,40)}$. Another meta-analysis and systematic review demonstrated that increased tomato consumption was inversely associated with prostate cancer risk ${ }^{(38)}$. However, these observations were not confirmed by other investigators ${ }^{(12-16)}$. In another published meta-analysis report, which pooled fifteen prospective cohorts, tomato consumption was not associated with a reduced risk of prostate cancer ${ }^{(16)}$. Dietary intake of lycopene as well as serum lycopene level showed an inverse association with the risk of cervical intraepithelial neoplasia in another study ${ }^{(41)}$. Zu et al. pooled the results of 49898 male health professionals from 1986 to 2010 and found a positive relationship between dietary lycopene intake and plasma lycopene level, with a mean plasma lycopene level of $934.5 \mathrm{~mol} / 1$ in the highest quintile of lycopene intake ( $n$ 1200), and concluded that higher lycopene intake was inversely associated with total prostate cancer and more strongly with lethal prostate cancer $^{(42)}$. In fact, a higher lycopene intake was associated with biomarkers in the cancer indicative of less angiogenic potential, thus probably reflecting less aggressive potential of the cancer ${ }^{(42)}$. In a cohort study, serum lycopene level was found to be inversely related to the risk of bladder cancer ${ }^{(43)}$. Of note, Giovannucci $^{(33)}$ reviewed seventy-two epidemiological studies; out of them, several studies have demonstrated an inverse relationship between intake of tomatoes or plasma lycopene level and cancer ${ }^{(33)}$. None of the studies analysed indicated there was an increased risk of cancer ${ }^{(33)}$. It appears that cancer risk is inversely associated with lycopene and tomato status, which can be improved by dietary sources rich in lycopene and tomato as well as through supplements ${ }^{(33)}$.

There are several proposed mechanisms for the protective action of lycopene in cancer prevention. Lycopene exerts strong antioxidant capacity because it contains many double-conjugated bonds: eleven conjugated double bonds and two unconjugated double bonds, which explains why lycopene can quench singlet oxygen and efficiently scavenge free radicals ${ }^{(44)}$. Lycopene is therefore a potent antioxidant, estimated to be twice as effective as $\beta$-carotene. Lycopene can potentially eliminate or neutralize reactive oxygen species, which is important in cancer chemoprevention since free radicals can facilitate carcinogenesis-related processes by means of the oxidation of cellular biomolecules. In addition, lycopene may exert its effect though the regulation of the antioxidant response element ${ }^{(45)}$ and can protect against oxidative stress, reactive oxygen species and other electrophilic species ${ }^{(44)}$. These properties of lycopene potentially protect macromolecules, such as carbohydrates, proteins, lipids and DNA, from oxidation and consequential carcinogenesis ${ }^{(44)}$, suggesting a possible inverse association between lycopene consumption and the risk of cancer. But apart from antioxidant capacity, other mechanisms contribute to cancer prevention, including immune modulation, hormone and growth factor signalling, regulatory mechanisms of cell-cycle progression, cell differentiation and apoptosis. In fact, the existence of this variety of mechanisms has led to the proposal that the initial effect of carotenoids should involve modulation 
of transcription ${ }^{(46)}$. Yang et al. reported that lycopene could suppress the proliferation of androgendependent human prostate tumour cells (LNCaP) through activation of the PPAR $\gamma-\mathrm{LXR} \alpha-\mathrm{ABCA} 1$ pathway ${ }^{(47)}$. Elgass et al. found that lycopene could also inhibit the cell adhesion and migration properties in androgen-independent prostate cancer cells (PC3 and DU145) ${ }^{(48)}$. In in vivo studies, dietary tomato and lycopene could have an influence on androgen signalling- and carcinogenesis-related gene expression during early prostate carcinogenesis in transgenic adenocarcinoma of the mouse prostate (TRAMP) mice ${ }^{(49)}$.

\section{Study strengths and limitations}

Our study has several strengths and some limitations. Because of the prospective design, the misclassification of exposure, which is unavoidable in dietary assessments, is most likely non-differential. Although we included several major potential confounders of lifestyle, clinical and dietary factors in the multivariable analysis, residual or unmeasured confounding might still exist. Furthermore, since this is an observational study, we cannot establish causality between tomato/lycopene consumption and our outcomes. In clinical trials, maintaining high adherence to a dietary intervention for a long time is typically difficult, in part because of dietary changes contradicting participants' long-term dietary preferences. Hence, poor adherence may dilute the true effect of an intervention. It is also important to bear in mind that the preparation method is important for determining the association between tomato (raw or cooked) consumption and risk of mortality ${ }^{(40)}$. Boiling is assumed to be the healthiest preparation method. Roasted and fried tomato are carriers of salt and fats, and acrylamide formation may be an additional problem in tomatoes cooked at temperatures above $120^{\circ} \mathrm{C}^{(51)}$. However, we did not have information on the way of preparation in the current analysis, and thus prospective studies focusing on this matter are needed. As the data collection was performed on all days of the week throughout the year in NHANES, the potential for day-specific information bias is very $\mathrm{low}^{(52,53)}$. We have evaluated the dietary lycopene intake, while plasma level of lycopene may could give us more valid results; but it has been reported that intake of lycopene has been associated with higher lycopene level in plasma, hence dietary also could be good marker of plasma level of lycopene ${ }^{(54)}$. Finally, we think it is important for future studies to assess the link of tomato/ lycopene consumption with each cancer type separately; we did not have information on this, so we could not perform such an analysis.

The major strength of our study is the large multi-ethnic US population-based sample of adults, making the results generalizable to the US population. In addition, there was standardized assessment of lycopene and tomato intake throughout the participating sites. The validity of the results is bolstered by working on both tomato and lycopene, as main compound of tomato, which is another study strength.

The present study has some important clinical and public health implications. A comprehensive understanding of the impact of diet, here particularly for tomato and lycopene consumption, and its impact on cancer mortality is a necessary and important step towards public health policy making and raising public awareness. Our study provides a comprehensive snapshot of a protective role of tomato and lycopene in cancer mortality at the national level that might be generalizable to the population level. By support of our results, we would like to encourage people to promote tomato consumption to achieve protective levels observed in highest tertile (T3), which seems to be relatively easy.

\section{Conclusion}

In conclusion, taken together, the beneficial results of lycopene and tomato consumption on cancer mortality provide reasonable and consistent evidence supporting the health benefits and important role of tomato products and lycopene as part of a healthy diet. These results are important for policy makers, contributing to increased public awareness about the role of the diet on health and the controversy regarding lycopene and tomato.

\section{Acknowledgements}

Financial support: This research received no specific grant from any funding agency in the public, commercial or notfor-profit sectors. Conflict of interest: The authors have no conflict of interest to declare. Authorship: M.M. contributed with study conception, data analysis and interpretation, and drafting the manuscript. M.B. and G.A.F. contributed with data interpretation and critical revision of the manuscript. Ethics of buman subject participation: This study was conducted according to the guidelines laid down in the Declaration of Helsinki and all procedures involving human subjects were approved by the Centers for Disease Control and Prevention. Written informed consent was obtained from all subjects.

\section{References}

1. American Cancer Society (2018) Cancer Facts \& Figures 2018. https://www.cancer.org/research/cancer-facts-statistics/allcancer-facts-figures/cancer-facts-figures-2018.html (accessed November 2019).

2. Slattery ML, Benson J, Curtin K et al. (2000) Carotenoids and colon cancer. Am J Clin Nutr 71, 575-582.

3. Giovannucci E, Stampfer MJ, Colditz G et al. (1992) Relationship of diet to risk of colorectal adenoma in men. J Natl Cancer Inst 84, 91-98. 
4. de Kok TM, de Waard P, Wilms LC et al. (2010) Antioxidative and antigenotoxic properties of vegetables and dietary phytochemicals: the value of genomics biomarkers in molecular epidemiology. Mol Nutr Food Res 54, 208-217.

5. Bao B, Azmi AS, Ali S et al. (2012) The biological kinship of hypoxia with CSC and EMT and their relationship with deregulated expression of miRNAs and tumor aggressiveness. Biochim Biophys Acta 1826, 272-296.

6. Sarkar FH, Li Y, Wang Z et al. (2010) The role of nutraceuticals in the regulation of Wnt and Hedgehog signaling in cancer. Cancer Metastasis Rev 29, 383-394.

7. Cheng HM, Koutsidis G, Lodge JK et al. (2017) Tomato and lycopene supplementation and cardiovascular risk factors: a systematic review and meta-analysis. Atherosclerosis 257, 100-108.

8. Banach M, Jankowski P, Jozwiak J et al. (2017) PoLA/CFPiP/ PCS guidelines for the management of dyslipidaemias for family physicians 2016. Arch Med Sci 13, 1-45.

9. Tan HL, Thomas-Ahner JM, Grainger EM et al. (2010) Tomato-based food products for prostate cancer prevention: what have we learned? Cancer Metastasis Rev 29, 553-568.

10. Dias MC, Vieiralves NF, Gomes MI et al. (2010) Effects of lycopene, synbiotic and their association on early biomarkers of rat colon carcinogenesis. Food Chem Toxicol 48, 772-780.

11. Palozza P, Colangelo M, Simone R et al. (2010) Lycopene induces cell growth inhibition by altering mevalonate pathway and Ras signaling in cancer cell lines. Carcinogenesis 31, 1813-1821.

12. Potischman N, McCulloch CE, Byers T et al. (1990) Breast cancer and dietary and plasma concentrations of carotenoids and vitamin A. Am J Clin Nutr 52, 909-915.

13. Jarvinen R, Knekt P, Seppanen R et al. (1997) Diet and breast cancer risk in a cohort of Finnish women. Cancer Lett 114 251-253.

14. Zhang S, Tang G, Russell RM et al. (1997) Measurement of retinoids and carotenoids in breast adipose tissue and a comparison of concentrations in breast cancer cases and control subjects. Am J Clin Nutr 66, 626-632.

15. Chen J, Song Y \& Zhang L (2013) Lycopene/tomato consumption and the risk of prostate cancer: a systematic review and meta-analysis of prospective studies. J Nutr Sci Vitaminol (Tokyo) 59, 213-223.

16. Petimar J, Wilson KM, Wu K et al. (2017) A pooled analysis of 15 prospective cohort studies on the association between fruit, vegetable, and mature bean consumption and risk of prostate cancer. Cancer Epidemiol Biomarkers Prev 26, 1276-1287.

17. Tighe P, Duthie G, Vaughan N et al. (2010) Effect of increased consumption of whole-grain foods on blood pressure and other cardiovascular risk markers in healthy middle-aged persons: a randomized controlled trial. Am J Clin Nutr $\mathbf{9 2}$, 733-740.

18. Mazidi M \& Kengne AP (2018) Higher adherence to plantbased diets are associated with lower likelihood of fatty liver. Clin Nutr 38, 1672-1677.

19. Centers for Disease Control and Prevention, National Center for Health Statistics (2019) National Health and Nutrition Examination Survey. http://www.cdc.gov/nchs/nhanes. htm (accessed November 2019).

20. Needham BL, Adler N \& Gregorich S et al. (2013) Socioeconomic status, health behavior, and leukocyte telomere length in the National Health and Nutrition Examination Survey, 1999-2002. Soc Sci Med 85, 1-8.

21. Mazidi M, Michos ED \& Banach M (2017) The association of telomere length and serum 25-hydroxyvitamin D levels in US adults: the National Health and Nutrition Examination Survey. Arch Med Sci 13, 61-65.

22. Centers for Disease Control and Prevention, National Center for Health Statistics (2011) Laboratory Procedure Manual:
C-Reactive Protein. http://www.cdc.gov/NCHS/data/ nhanes/nhanes_09_10/CRP_F_met.pdf (accessed August 2017).

23. Ahluwalia N, Andreeva VA, Kesse-Guyot E et al. (2013) Dietary patterns, inflammation and the metabolic syndrome. Diabetes Metab 39, 99-110.

24. Ahluwalia N, Dwyer J, Terry A et al. (2008) Update on NHANES dietary data: focus on collection, release, analytical considerations, and uses to inform public policy. Adv Nutr $\mathbf{7}$, 121-134.

25. Moshfegh AJ, Rhodes DG, Baer DJ et al. (2008) The US Department of Agriculture automated multiple-pass method reduces bias in the collection of energy intakes. Am J Clin Nutr 88, 324-332.

26. Levey AS, Stevens LA, Schmid CH et al. (2009) A new equation to estimate glomerular filtration rate. Ann Intern Med 150, 604-612.

27. Expert Committee on the Diagnosis and Classification of Diabetes Mellitus (1997) Report of the expert committee on the diagnosis and classification of diabetes mellitus. Diabetes Care 20, 1183-1197.

28. Nwankwo T, Yoon SS, Burt V et al. (2013) Hypertension among adults in the United States: National Health and Nutrition Examination Survey, 2011-2012. NCHS Data Brief issue 133, 1-8.

29. Centers for Disease Control and Prevention, National Center for Health Statistics (2019) NCHS Data Linkage. https:// www.cdc.gov/nchs/data-linkage/mortality.htm (accessed November 2019).

30. US Department of Health and Human Services, Centers for Disease Control and Prevention, National Center for Health Statistics (2013) National Health and Nutrition Examination Survey: Analytic Guidelines, 1999-2010. https://www.cdc. gov/nchs/data/series/sr_02/sr02_161.pdf (accessed January 2018).

31. Mazidi M, Wong ND, Katsiki N et al. (2017) Dietary patterns, plasma vitamins and trans fatty acids are associated with peripheral artery disease. Lipids Health Dis 16, 254.

32. Fine JP \& Robert JG (1999) A proportional hazards model for the subdistribution of a competing risk. J Am Stat Assoc 94, 496-509.

33. Giovannucci E (1999) Tomatoes, tomato-based products, lycopene, and cancer: review of the epidemiologic literature. J Natl Cancer Inst 91, 317-331.

34. Franceschi S, Bidoli E, La Vecchia C et al. (1994) Tomatoes and risk of digestive-tract cancers. Int J Cancer 59, 181-184.

35. Mein JR, Lian F \& Wang XD (2008) Biological activity of lycopene metabolites: implications for cancer prevention. Nutr Rev 66, 667-683.

36. Gann PH, Ma J, Giovannucci E et al. (1999) Lower prostate cancer risk in men with elevated plasma lycopene levels: results of a prospective analysis. Cancer Res 59, 1225-1230.

37. Rao AV, Fleshner N \& Agarwal S (1999) Serum and tissue lycopene and biomarkers of oxidation in prostate cancer patients: a case-control study. Nutr Cancer 33, 159-164.

38. Rowles JL 3rd, Ranard KM, Applegate CC et al. (2018) Processed and raw tomato consumption and risk of prostate cancer: a systematic review and dose-response meta-analysis. Prostate Cancer Prostatic Dis 21, 319-336.

39. Diallo A, Deschasaux M, Galan P et al. (2016) Associations between fruit, vegetable and legume intakes and prostate cancer risk: results from the prospective Supplementation en Vitamines et Mineraux Antioxydants (SU.VI.MAX) cohort. Br J Nutr 115, 1579-1585.

40. Graff RE, Pettersson A, Lis RT et al. (2016) Dietary lycopene intake and risk of prostate cancer defined by ERG protein expression. Am J Clin Nutr 103, 851-860.

41. VanEenwyk J, Davis FG \& Bowen PE (1991) Dietary and serum carotenoids and cervical intraepithelial neoplasia. Int J Cancer 48, 34-38. 
42. Zu K, Mucci L, Rosner BA et al. (2014) Dietary lycopene, angiogenesis, and prostate cancer: a prospective study in the prostate-specific antigen era. J Natl Cancer Inst 106, dit430.

43. Helzlsouer KJ, Comstock GW \& Morris JS (1989) Selenium, lycopene, alpha-tocopherol, beta-carotene, retinol, and subsequent bladder cancer. Cancer Res 49, 6144-6148.

44. Marti R, Rosello S \& Cebolla-Cornejo J (2016) Tomato as a source of carotenoids and polyphenols targeted to cancer prevention. Cancers 8, E58.

45. Trejo-Solis C, Pedraza-Chaverri J, Torres-Ramos M et al (2013) Multiple molecular and cellular mechanisms of action of lycopene in cancer inhibition. Evid Based Complement Altern Med 2013, 705121.

46. Tanaka T, Shnimizu M \& Moriwaki H (2012) Cancer chemoprevention by carotenoids. Molecules 17, 3202-3242.

47. Yang CM, Lu IH, Chen HY et al. (2012) Lycopene inhibits the proliferation of androgen-dependent human prostate tumor cells through activation of PPARgamma-LXRalpha-ABCA1 pathway. J Nutr Biochem 23, 8-17.

48. Elgass S, Cooper A \& Chopra M (2014) Lycopene treatment of prostate cancer cell lines inhibits adhesion and migration properties of the cells. Int J Med Sci 11, 948-954.

49. Wan L, Tan HL, Thomas-Ahner JM et al. (2014) Dietary tomato and lycopene impact androgen signaling- and carcinogenesis-related gene expression during early TRAMP prostate carcinogenesis. Cancer Prev Res (Phila) 7, 1228-1239.

50. Etminan M, Takkouche B \& Caamano-Isorna F (2004) The role of tomato products and lycopene in the prevention of prostate cancer: a meta-analysis of observational studies. Cancer Epidemiol Biomarkers Prev 13, 340-345.

51. Mottram DS, Wedzicha BL \& Dodson AT (2002) Acrylamide is formed in the Maillard reaction. Nature 419, 448-449.

52. Tooze JA, Midthune D, Dodd KW et al. (2006) A new statistical method for estimating the usual intake of episodically consumed foods with application to their distribution. J Am Diet Assoc 106, 1575-1587.

53. Guenther PM, Ding EL \& Rimm EB (2013) Alcoholic beverage consumption by adults compared to dietary guidelines: results of the National Health and Nutrition Examination Survey, 2009-2010. J Acad Nutr Diet 113, 546-550.

54. Gustin DM, Rodvold KA, Sosman JA et al. (2004) Single-dose pharmacokinetic study of lycopene delivered in a welldefined food-based lycopene delivery system (tomato paste-oil mixture) in healthy adult male subjects. Cancer Epidemiol Biomarkers Prev 13, 850-860. 